\title{
La categorización de algunos cuantificadores discursivos de medida en la lengua aimara
} The categorization of some discourse quantifiers extent Aymara language

\section{Karina Salazar Tantalean}

kari.salazart@gmail.com

\section{Silvia Sinchitullo Rosales} silvispaty@hotmail.com

Universidad Nacional Mayor de San Marcos, Lima, Perú

\section{RESUMEN}

Esta investigación tiene como objetivo fundamental describir la categorización semántica de algunos cuantificadores discursivos de medida que los aimarahablantes emplean en el discurso comercial, desde el enfoque de la semántica cognitiva. Los hablantes de la lengua aimara categorizan los cuantificadores discursivos de medida sobre la base de la naturaleza física de los entes que cuantifican. En ese sentido, encontramos cuantificadores como jachi («lo que cabe en un puño»), jarphi («lo que cabe en una pollera»), etc. Dichas expresiones, además, son formas lingüísticas categorizadas en función de la experiencia del hablante, que se enmarca dentro del entorno cultural del pueblo aimara.

\section{PALABRAS CLAVE}

Categorización, cuantificación discursiva, semántica cognitiva

\section{ABSTRACT}

This investigation has as main objective to describe the semantic categorization of some discourse quantifiers as the Aymara speakers used in commercial speech, from the perspective of Cognitive Semantics. The speakers of the Aymara language categorized as discursive quantizers based on the physical nature of the entities that quantify. In that sense, we found quantifiers as jachi (what fits in a fist), jarphi (what fits in a skirt), etc. These expressions linguistic forms are categorized according to the speaker's experience, which is part of the cultural environment of the Aymara people. 


\section{KEYWORDS}

Categorization, quantification discursive, cognitive semantics

\section{Introducción}

Cada lengua tiene una manera particular de categorizar los entes que existen dentro de la realidad, de acuerdo con la experiencia que los hablantes adquieren al momento de interactuar con el medio donde viven. Cuando hablamos de categorizar, hacemos referencia básicamente, según Cuenca (1999, p. 32), a «la organización de la información obtenida a partir de la aprehensión de la realidad».

Precisamente, una manera de ordenar parte de la realidad la encontramos en las formas de cuantificar los entes concretos de una cultura. Los hablantes de la lengua aimara categorizan los cuantificadores discursivos basados en la naturaleza física de los entes que pretenden cuantificar.

En ese sentido, las formas discursivas como jachi («lo que cabe en un puño») y jarphi («lo que cabe en una pollera») se realizan en contextos de intercambio comercial. Dichas expresiones, para los aimaras, son formas lingüísticas que tienen inmersa la noción de cuantificación.

Estas formas de cuantificar se relacionan con la experiencia que ellos tienen en su vida cotidiana y que se desarrolla dentro de su entorno cultural.

Debemos mencionar también que los aimarahablantes solo practican esta forma de cuantificar en algunas comunidades, como las ferias de los domingos, donde aún se realiza el intercambio de productos. A dicho intercambio se le denomina chala, comúnmente conocido como trueque.

\section{Metodología}

Los datos para el análisis del presente estudio han sido obtenidos en el proyecto de investigación sobre la elaboración del Manual de enseñanza aimara desde un enfoque integral realizado en 2010, cuyo responsable fue el profesor Felipe Huayhua. Este proyecto se realizó en los distritos de Ilave, Conima, Ninantaya y Chucuito.

Nuestro trabajo consistió, primero, en observar el intercambio comercial que se realizaba en algunas ferias. Luego, realizamos una encuesta a 20 hablantes de la lengua aimara de estos distritos. Posteriormente, revisamos diccionarios en donde se encontraban registrados algunos de estos cuantificadores discursivos de medida, con el fin de conocer sus diversos usos. 


\section{Marco teórico: semántica cognitiva}

Según Cuenca (1999), la categorización es un proceso cognitivo que consiste en organizar todos los entes que se extraen de la realidad. Este mecanismo de organización nos permite simplificar la infinitud de entidades que existen dentro de la realidad. Asimismo, afirma que «la categorización se puede definir como un proceso mental de clasificación cuyo producto son las categorías cognitivas»; además, este mecanismo «fundamenta los procesos de comprensión y producción lingüística» (p. 32).

Dicha categorización se realiza a partir de dos procedimientos fundamentales que se complementan: la generalización o abstracción y la discriminación. Por un lado, generalizar consiste en no considerar las diferencias entre entidades con el fin de agruparlas según sus semejanzas, mientras que discriminar implica establecer los rasgos que diferencian dos o más entidades con el objetivo de no confundirlas.

Cuenca $(1999$, p. 32) afirma que estos procesos mentales son importantes para la estructuración conceptual y la generación del pensamiento:

Si no generalizáramos, no podríamos sobrepasar el nivel de las entidades individuales y la realidad sería caótica y constantemente nueva, de forma que no podríamos llegar a una estructuración conceptual. Si no discrimináramos, todo sería uno y tampoco habría pensamiento.

Además, Cuenca afirma que las categorías se asocian con un prototipo, esto es, una imagen mental que se forma a partir de la interacción de una serie de atributos característicos. Dicha asociación permite hablar de buenos ejemplares (prototipos) y de malos ejemplares (miembros periféricos). Estos últimos marcan fronteras difusas respecto a otras categorías.

Para Kleiber (1995), la categorización toma como base el grado de similitud con el ejemplar más idóneo. Un prototipo, es pues, el ejemplar típico de una categoría; los demás son equiparados a esta categoría por su semejanza con el prototipo. Cuanto más se parezca al prototipo el elemento que categoricemos, por ejemplo, un gorrión para la categoría de los pájaros, o manzanas para la categoría fruta, con más claridad se verá su pertenencia a la categoría que le concierne.

Según Cifuentes (1994), la categorización no tiene realidad existencial, sino funcional; afirma que se trata de una construcción metodológica $y$, por ello, su poder descriptivo es fundamental. De esa manera, al aplicarse a elementos no idénticos se gana precisión. Por otro lado, continúa Cifuentes, no todos los elementos relacionantes tienen las mismas características; sin embargo, todos cumplen el concepto funcional de los entes que se pretende describir. 


\subsection{Cuantificar}

La Real Academia Española define el término cuantificar como «expresar numéricamente una magnitud». Por ejemplo, al medir una propiedad física como 1,35 metros o pesar 90 kilos, estamos valorando la extensión cuantitativa o numérica de una propiedad, o estimando el número de personas que la poseen. De esa manera estamos emitiendo juicios valorativos (López, 1999).

\subsection{Cuantificadores discursivos}

Denominamos cuantificadores discursivos de medida aquellas formas lingüísticas que expresan conceptos de cuantificación en un determinado discurso. En la presente investigación, hacemos referencia a los cuantificadores que los hablantes aimaras hacen uso en un discurso cotidiano (no especializado que parte de una necesidad cotidiana de intercambio y de labores que a diario realizan).

\section{Análisis}

Lo que presentaremos a continuación es el corpus que hemos seleccionado para el desarrollo de nuestra investigación. Realizaremos una breve descripción del uso pragmático de estos cuantificadores. Luego, describiremos cómo los hablantes de la lengua aimara categorizan estos entes conceptuales, de acuerdo con la interacción y aprehensión que ellos han ejercido de su medio cultural.

Cabe señalar que esta forma de cuantificar es propia de esta cultura y que proviene de tiempos muy antiguos, cuando se realizaba intercambio de productos. A esto se denomina operación chala, es decir, de intercambio de productos, conocido como trueque. Dicho proceso se realizaba en los qhatu, que son ferias que se organizaban a diario en diferentes distritos. Estas se desarrollaban durante los días de semana en distritos como Ilave, Conima, Ninantaya, Chuquito, etc. Con el paso del tiempo y la occidentalizacion, poco a poco la costumbre fue disminuyendo; ahora se organizan generalmente los domingos.

La cuantificación señalada no se realiza en el corpus en todas las ferias, porque la mayoría ya conoce la manera de medir en castellano. Solo en lugares como Moho, provincia de Puno, y Pomata se mantienen estas formas de contabilizar. Así pues, los lugares donde se mantienen estas formas de medir presentan una menor influencia de la cultura occidental y mayor índice de pobreza.

Siguiendo la propuesta de la semántica cognitiva, la categorización de los cuantificadores discursivos estaría determinada de la siguiente manera. Los hablantes nativos de la lengua aimara categorizan sus cuantificadores 
discursivos sobre la base de la naturaleza de los objetos. Se generaliza, es decir, se obvian las diferencias entre los cuantificadores y los objetos involucrados para agruparlos según su semejanza.

Por ejemplo, en el caso de los cuantificadores jachi y phuxto, se establecen semejanzas entre ellos porque se emplean para medir productos pequeños, como cebada, habas, maíz, etc. A la vez, se discriminan rasgos diferenciales entre los cuantificadores y los objetos para realizar la categorización. Podemos observar que los cuantificadores como jarphi o t'imphi se emplean para cuantificar productos medianos como papa, camote, pan, etc. Además, jarphi se utiliza básicamente en la cosecha, mientras que t'imphi se emplea en otros contextos.

Los cuantificadores discursivos de medida, como luqa y wara, se agrupan según la extensión del objeto a cuantificar.

Los procesos cognitivos mencionados se realizan de forma simultánea para establecer dicha categorización.

Asimismo, los aimaras hablantes categorizan sus cuantificadores discursivos -en términos de Cifuentes - basados en la funcionalidad, es decir, de acuerdo con el uso que los objetos a ser cuantificados adquieren según las necesidades y las experiencias del pueblo aimara. En otras palabras, los categorizan según la realidad de su entorno cultural, a las necesidades de intercambio comercial que ellos practicaban y que aún siguen practicando en algunas comunidades.

Según la teoría, entendemos que el prototipo es la imagen mental que se forma en los hablantes respecto a una categoría. Esta imagen es considerada como el ejemplar prototípico, es decir, el modelo típico de una categoría que el hablante evoca en primera instancia.

Según nuestras encuestas realizadas, el elemento prototípico de los cuantificadores discursivos de medida es jach' ${ }^{1}{ }^{1}$, ya que el 70 por ciento de los encuestados lo mencionó como primera respuesta. Probablemente, esto se debe a que lo más común en estos intercambios comerciales (trueques) es que los aimaras realizan intercambios de cereales.

\subsection{Por la naturaleza física del objeto que cuantifican}

\subsubsection{Por lo que cabe en un ente referencial}

Esta forma de cuantificar se agrupa en torno a los rasgos de tamaño: los objetos que se cuantifican pueden ser diminutos, medianos o de naturaleza alargada.

1 Como mencionamos, realizamos una encuesta a 20 personas y entre las preguntas consignamos la siguiente: «¿Menciona un tipo de medida?». 
Para cuantificar objetos diminutos como cereales, se hace uso de los cuantificadores discursivos:

- Jach'i: lo que cabe en un puño. Esta medida se utiliza sobre todo para cereales.

Asimismo, Huayhua (2009, p. 109) define este cuantificador como «medida usual equivalente a un manojo o puñado».

- Phuxtu: lo que cabe en las dos manos. Esta manera de medir también se emplea especialmente para cereales.

Del mismo modo, Huayhua (2009, p. 182) define el término phuxtu como «llevar algo con las dos palmas de la mano».

Para cuantificar entes medianos como papa, camote, pan, piedras medianas, etc., los hablantes, en determinado contexto comunicativo, hacen uso de los siguientes cuantificadores discusivos:

- Jarphi: lo que cabe en la pollera². Esta medida se utiliza básicamente en la cosecha.

- T'imphi: lo que cabe en la pollera.

Huayhua (2009, p. 114) define a t'imphi como «parte delantera de la falda, regazo».

- Lak'u: lo que cabe en una manta.

- Inkuña: lo que cabe en una manta pequeña. Son tejidas por señoras que utilizan la palma de la mano para medir el tamaño del tejido. Hay tres tamaños de inkuña: pequeña, mediana y grande, y se usan para medir productos como papa, coca, etc. Son empleadas asimismo para exhibir productos medianos en las ferias. El término inkuña también puede ser definido como «mantel mediano y cuadrado, tejido de lana de oveja o llama, que usan para llevar fiambre, hay de diversos tamaños desde pequeño istalla ${ }^{3}$ hasta inkuña» Huayhua (2009, p. 103).

Para cuantificar entes grandes o de naturaleza alargada - como palos, totora, paja, etc.-, los aimaras utilizan determinados cuantificadores discursivos:

- Marqa: lo que cabe en una brazada (con los dos brazos). Esta medida se utiliza en productos de naturaleza alargada, como totora, paja, etc. El término marqa también es definido como «medida

2 Falda externa del vestido femenino, según la novena acepción para dicho término, propuesto por la Real Academia Española (2014).

3 Huayhua (2009) define este término como un tejido pequeño con el cual las mujeres llevan la coca en días festivos. Algunas istalla son parecidas a la taleguilla. 
de una brazada, todo lo que cabe entre los dos brazos» (Huayhua, 2009, p. 157).

- Sixi: lo que cabe en una estera rectangular y se envuelve como cilindro. La expresión lingüística sixi también se define como «troje hecho de totoras» (Huayhua, 2009, p. 214).

\subsubsection{Para establecer la extensión o medida longitudinal de un objeto}

Estos cuantificadores discursivos se utilizan para medir la extensión de un objeto (por ejemplo, para medir la longitud de lana, tela, soga, etc.):

- Luqa: es la distancia entre el índice de la mano derecha hasta el índice de la mano izquierda, con los brazos estirados. Se utiliza para medir la longitud de las sogas de lana de oveja o de llama y las sogas de paja. El término luqa ha sido definido asimismo como «medida equivalente a una brazada, comprendida desde el dedo medio de la mano derecha hasta la izquierda extendidos» (Huayhua, 2009, p. 147).

- Wara: es la distancia entre la mandíbula inferior y los dedos índice y pulgar de un brazo estirado, paralelo al piso horizontal. Se utiliza para medir soga, tela, etc.

El Ministerio de Educación (1983) también ha definido el término wara como una unidad de medida equivalente a la distancia generada entre la mandíbula inferior y los dedos índice y pulgar de un brazo estirado lateralmente, que sostiene una de las puntas de la tela o soga que se mide.

- Cchiya: es la distancia entre las puntas de los dedos pulgar y meñique estirados. Se utiliza para medir la longitud de la soga y la tela de corto tamaño.

Para el Ministerio de Educación (1983, p. 110), el término cchiya se refiere a «la distancia entre las puntas de los dedos pulgar y meñique estirados».

- Chilqui: es la distancia que se genera cuando una persona adulta da un paso, es decir, la longitud formada entre un pie y el otro al dar el paso. Se utiliza para la distribución de terreno.

El término chilqui «es la distancia entre dos pies de una persona adulta al dar un paso» (Ministerio de Educación, 1983, p. 113).

\section{Conclusiones}

Los cuantificadores discursivos de medida son formas lingüísticas que expresan el concepto de cuantificación en un determinado uso discursivo.

Estos cuantificadores discursivos de medida forman parte del legado ancestral de la cultura aimara, cuyo uso, actualmente, se está perdiendo por la influencia de las formas de medir de la cultura occidental. Solo en algunas comunidades se mantienen estas formas de cuantificar. 
Los aimaras hablantes categorizan los cuantificadores discursivos de medida de acuerdo con la naturaleza física de los objetos que pretenden cuantificar y lo que cabe en un determinado ente referencial, o según la extensión o medida longitudinal del objeto a cuantificar.

Esta categorización se realiza sobre la base de los rasgos similares establecidos entre un producto y otro. Por ejemplo, phuxtu y jach'i son formas lingüísticas que cuantifican objetos diminutos. Por su parte, los cuantificadores discursivos de medida, como luqa y wara, se agrupan según la extensión del objeto a cuantificar.

Los aimaras hablantes categorizan sus cuantificadores discursivos en función del uso que los objetos a ser cuantificados adquieren, según las necesidades del pueblo aimara. También lo hacen basándose en la realidad de su entorno cultural y en las prácticas de intercambio comercial que aún existen en algunas comunidades.

El prototipo de los cuantificadores discursivos de medida es jach'i. 


\section{REFERENCIAS BIBLIOGRÁFICAS}

Cifuentes, J. (1994). Gramática cognitiva. Fundamentos críticos. Madrid: Eudema.

Cuenca, M. y Hilferty, J. (1999). Introducción a la lingüística cognitiva. Barcelona: Editorial Ariel.

Huayhua, F. (2009). Diccionario bilingüe polilectal aimara-castellano casteIlano-aimara. Lima: Fondo Editorial de la Universidad Nacional Mayor de San Marcos.

Kleiber, G. (1995). La semántica de los prototipos: categoría y sentido léxico. Madrid: Editorial Visor.

López, H. (1999). La interpretación de los cuantificadores. Aspectos sintácticos y semánticos. Madrid: Editorial Visor.

Ministerio de Educación (1983). Numeración, algoritmos y aplicación de relaciones numéricas y geométricas en las comunidades rurales de Puno. Lima: Ministerio de Educación.

Real Academia Española (2014). Pollera. Recuperado de http://lema.rae. es/drae/?val=pollera 